\title{
Rôle d'Hylobius abietis (L) (Col, Curculionidae) dans le transport de Leptographium procerum (Kendr) Wingf et son inoculation au pin sylvestre
}

\author{
D Piou \\ ENGREF, Arboretum national des Barres, 45290 Nogent-sur-Vernisson, France
}

(Reçu le 17 janvier 1992; accepté le 28 décembre 1992)

\begin{abstract}
Résumé - On a étudié la fréquence des associations entre Hylobius abietis et divers champignons, dans 4 parcelles de la forêt d'Orléans en 1989 et 1990, d'une part, et dans plusieurs localités des Vosges et du Massif central en 1990, d'autre part. Seul Leptographium procerum se montrait véritablement lié à l'hylobe. La fréquence de contamination de l'insecte par ce champignon variait fortement d'un site à l'autre. Elle était également fonction, semble-t-il, de la date des prélèvements. Les émergents immatures, mâles et femelles, pouvaient également être contaminés par $L$ procerum, mais cette contamination ne semblait pas avoir d'effet sur leur poids. $L$ procerum a, par ailleurs, été isolé de plants de pin sylvestre (Pinus sylvestris $\mathrm{L}$ ) morts à la suite de morsures de maturation de l'insecte. Des inoculations de $L$ procerum au niveau de larges blessures, pratiquées artificiellement sur des plants de pin sylvestre et imitant les dégâts d'hylobe, ont provoqué la mort de tous les plants. Leur système racinaire et, à un moindre degré, leur partie aérienne étaient envahis par le champignon. Les plants témoins, blessés de la même façon mais non inoculés, ont tous survécu. Ces résultats suggèrent que $L$ procerum pourrait être en partie responsable de la mortalité des plants observée dans les reboisements attaqués par l'hylobe.
\end{abstract}

Hylobius abietis / Leptographium procerum / relation insecte-champignon / mortalité de plants

Summary - Possible role of Hylobius abietis (L) in the transport of Leptographium procerum (Kendr) Wingf and in the infection of Scots pine. The aim of this study was to determine the fungus species liable to be conveyed by the pine weevil Hylobius abietis, considered to be the main pest of coniferous reafforestations. The study concentrated mainly on the frequency variations of each isolated fungus in 4 Scots pine stands in the Orleaans forest (centre of France) in 1989 and 1990, and in 2 mountainous sites (Vosges and Massif Central) in 1990. Leptographium procerum alone - because of its relatively frequent association with the adult insects during the 2 years and in all trapping sites - appeared to have a link with the pine weevil (table I). The contamination frequency due to this fungus revealed important variations from one site to another (figs 1,2), but the contamination also seemed to depend on the collection period during the year (fig 3). The immature emergents, both male and female, could also be contaminated (table II). This contamination was probably linked to the development of $\mathrm{L}$ procerum in its saprophytic phase. The contamination by $\mathrm{L}$ procerum 
did not seem to have any effect on the weight of the emergents. The fungus had also been isolated from dead Scots pine (Pinus sylvestris $L$ ) seedlings, after feeding damage by the pine weevil. Inoculations at the collar with patches of agar cultures on 12 seedlings with artificial substantial wounding revealed high mortality whereas the wounds alone did not lead to mortality. In that case, $L$ procerum developed mainly in the root part of the seedlings and rarely in the aerial part (fig 4). These experiments suggest that $L$ procerum could increase the mortality rate of seedlings after partial feeding damage by the pine weevil.

Hylobius abietis / Leptographium procerum / insect-fungus relationship / seedling mortality

\section{INTRODUCTION}

Hylobius abietis est le principal représentant européen du genre Hylobius. Durant la phase sous-corticale de son cycle, il ne s'attaque qu'à des arbres très affaiblis ou à des souches fraîches, mais il effectue son repas de maturation sur des rameaux ou des plants. Dans ce dernier cas, les dégâts peuvent être importants et $H$ abietis est considéré comme le plus important ravageur des reboisements de résineux en Europe (Eidmann, 1974; Langström, 1982; Heritage et al, 1989). Pour certains insectes sous-corticaux, le repas de maturation est l'occasion d'inoculer à un arbre sain des micro-organismes pathogènes; les scolytides de l'orme inoculant Ophiostoma ulmi (Lanier et Peacock, 1981), le cérambycide Monochamus alternatus inoculant le nématode Bursaphelenchus xylophlus (de Guiran, 1990) sont des exemples bien connus. En Europe, si de nombreux travaux ont été consacrés aux champignons véhiculés par les scolytides (Francke-Grossman, 1967; Lieutier et al, 1989; Furniss et al, 1990), pratiquement aucune recherche n'a été entreprise sur les champignons véhiculés par les curculionides. Or, compte tenu du comportement de maturation d' $H$ abietis, l'inoculation de champignons à des plants n'est pas à exclure. Nous nous sommes intéressé aux éventuels champignons associés à cet insecte, et à ceux présents à proximité de morsures sur des plants attaqués. Le rôle de ces champignons dans la mortalité des plants sera discuté.

\section{MATÉRIELS ET MÉTHODES}

\section{Piégeage des hylobes en 1989}

Les insectes ont été capturés dans 4 parcelles de pin sylvestre (Pinus sylvestris $\mathrm{L}$ ) de la forêt d'Orléans, dans le Centre de la France, au printemps 1989. Deux parcelles (B140 et B339) portaient des arbres âgés de 40 à 50 ans, dont quelques dépérissants répartis en taches. Les symptômes de ce dépérissement ont été décrits par Piou et Lieutier (1989). Les 2 autres parcelles étaient des coupes à blanc de pins sains, réalisées durant l'hiver 1988-1989 (T325 et T376).

Des rondins frais de $1 \mathrm{~m}$ de longueur de pin sylvestre ont été réalisés à partir d'arbres sains. Deux fragments de liber ont été prélevés stérilement sur chaque rondin. Les rondins ont été écorcés suivant une génératrice d'environ 3-4 $\mathrm{cm}$ de largeur, sur toute leur longueur. Dans chacune des 4 parcelles choisies, 10 rondins ainsi préparés ont été à demi enterrés, la face écorcée contre le sol, puis recouverts d'humus et de fougères. Les rondins devenant moins attractifs avec le temps (Langström, 1982), ils ont tous été remplacés par des rondins frais identiquement préparés, au moins une fois durant la période de piégeage. Les rondins ont été déterrés et retournés chaque semaine entre le $1^{\text {er }}$ mars et le 6 juillet 1989. Les hylobes présents ont été prélevés à l'aide d'une pince trempée dans l'alcool puis passée à la flamme. Chaque insecte a ensuite été placé dans un tube stérile. Ramenés au laboratoire, les tubes ont été placés à $4^{\circ} \mathrm{C}$ jusqu'à utilisation. 


\section{Piégeage des hylobes en 1990}

Les piégeages d'hylobe ont été poursuivis en 1990, au printemps (du 19 avril au 15 mai) et en été (du 16 au 20 août), sur 3 sites :

- la forêt d'Orléans, dans les 4 parcelles de pin sylvestre utilisées en 1989;

- une coupe à blanc d'épicéa commun (Picea abies Karst) située à $200 \mathrm{~km}$ au sud de la forêt d'Orléans, dans le Massif central (Creuse) à 600 m d'altitude;

- 7 reboisements de la région de Bruyères (Vosges) situés à $350 \mathrm{~km}$ à l'est de la forêt d'Orléans, entre 360 et $630 \mathrm{~m}$ d'altitude, après coupes à blanc de peuplements mélangés pin sylvestre - sapin (Abies alba Mill) pour les prélèvements de printemps, et après une coupe à blanc d'épicéa commun pour ceux d'été.

Sur les 2 premiers sites, les piégeages ont été réalisés selon la méthode employée en 1989. Dans les Vosges, les insectes ont été prélevés stérilement sur plants, pendant leur repas de maturation.

\section{Obtention d'hylobes émergents}

Seulement 10 des rondins installés en 1989 en forêt d'Orléans avaient encore une écorce intacte le 20 avril 1990, date à laquelle ils ont été placés en éclosoir au laboratoire. Huit des rondins mis en place dans les mêmes parcelles, le 15 mars 1990 , ont été placés en éclosoir au laboratoire le 16 juin 1990 pour la moitié et le 20 août 1990 pour l'autre moitié. Les insectes émergents ont été prélevés stérilement au fur et à mesure de leur sortie et placés individuellement en tube stérile, à $4^{\circ} \mathrm{C}$ jusqu'à utilisation. Avant mise en éclosoir, une rondelle de bois de $5 \mathrm{~cm}$ de large environ a été prélevée au milieu de chaque rondin, puis placée en chambre humide.

\section{Isolement de champignons à partir des insectes}

Les insectes ont été extraits stérilement des tubes et placés individuellement en boîte de Pétri sur un mélange malt (33 g/l)-agar, addition- né de $500 \mathrm{mg} / \mathrm{l}$ de cycloheximide après l'autoclavage du milieu afin de limiter la croissance des bactéries (McCall et Merril, 1980). La croissance des espèces appartenant aux genres Ophiostoma, Europhium sp et aux anamorphes correspondantes n'est pas affectée par cet antibiotique (Harrington, 1981). Les insectes ont été laissés libres de leurs déplacements dans la boîte pendant 5 min environ, puis la boîte contenant l'insecte a été agitée manuellement pendant $1 \mathrm{~min}$. Les insectes émergents ont été sexés et pesés après cette phase d'isolement.

Les fragments de liber prélevés sur les rondins avant leur dépôt en forêt ont été placés sur un milieu malt-agar.

\section{Isolements de champignons à partir de plants attaqués par l'hylobe}

En parcelle T325 de la forêt d'Orléans, 40 plants morts de pin sylvestre attaqués par l'insecte ont été arrachés en juillet 1990 et rapportés au laboratoire. Sur chaque plant, 5 rondelles de bois ont été prélevées au niveau des morsures de maturation pratiquées par $H$ abietis. Ces prélèvements ont été mis en culture sur le milieu malt-agar-cycloheximide.

\section{Inoculation sur plants du champignon le plus fréquermment associé à l'insecte : Leptographium procerum}

Vingt-quatre plants de 3 ans de pin sylvestre ont été placés en pot de 2 I en mars 1990. En octobre 1990, 8 blessures de $4 \mathrm{~mm}$ de diamètre ont été réalisées au collet de chaque plant, avec un emporte-pièce préalablement stérilisé. Afin de représenter les blessures effectuées par l'hylobe tout en évitant de provoquer une annélation circulaire, les blessures ont été réparties en quinconce sur 2 couronnes distantes de $2 \mathrm{~cm}$.

Des implants ont été prélevés en marge d'une culture monospore de $L$ procerum de 3 semaines développée sur malt-agar. Un implant a été déposé dans chaque blessure de 12 plants prélevés au hasard. La souche utilisée provenait d'une racine bleuie d'un pin sylvestre dominant et apparemment sain de 40 ans, situé en parcelle B140. En outre, une larve $\mathrm{d}^{\prime} \mathrm{H}$ abietis 
se développait à l'intérieur de cette racine. Sur les 12 pots restants, des implants de malt-agar ont été déposés au niveau de chaque blessure. La zone inoculée a été protégée par un coton humidifié à l'eau stérile maintenu par un film plastique. En mars 1991, le pourcentage de mortalité a été noté. Le réisolement du champignon a été tenté de part et d'autre de la zone d'inoculation, tous les $2,5 \mathrm{~cm}$ sur la tige principale, et à $5 \mathrm{~cm}, 10 \mathrm{~cm}$ et $15 \mathrm{~cm}$ du collet, sur la racine principale et sur les 2 plus grosses racines secondaires.

\section{Analyse des données}

Les intervalles de confiance des résultats ont été calculés au seuil de $95 \%$. Les fréquences relatives de contamination ont été comparées à l'aide du test $\mathrm{G}$ ajusté (Scherrer, 1984). Des analyses factorielles de variance ont permis de comparer le poids des insectes émergents en fonction de leur sexe, des rondins d'émergence et de la présence de champignons contaminants.

\section{RÉSULTATS}

\section{Champignons présents sur les adultes d'Hylobius abietis}

Quatre cent vingt-quatre insectes ont été piégés en forêt d'Orléans du 14 mars au 6 juillet 1989 , avec des différences notables de capture entre parcelles : moins de 80 insectes en T376 et B140, quasiment tous piégés après le 11 mai, une centaine d'insectes en T325 avec quelques prises avant le 11 mai et 172 insectes en B339 avec des prises importantes avant cette date. Cent quarante-cinq insectes seulement ont été capturés au total en 1990 dans les 3 sites de piégeage. Les pièges installés dans le Massif central ont été très peu performants, et aucun insecte n'y a été capturé en été.

Les fragments de liber prélevés sur les rondins-pièges avant leur installation n'ont permis de mettre en évidence que des saprophytes classiques (Penicillium, Trichoderma, Mucorales). Trois autres champignons ont été isolés à partir des adultes d'hylobe:

- Leptographium procerum (Kendr), Wingf;

- Pachnodium canum (Upadh \& Kendr) dont la forme parfaite est Ophiostoma canum (Münch), H \& $\mathrm{P}$ Syd, forme que nous n'avons jamais observée en culture;

- Ophiostoma piliferum (Fries), H \& P Syd.

Les pourcentages d'association de ces 3 champignons avec les adultes étaient très différents (tableau I). $O$ piliferum était l'espèce la moins fréquemment associée à $H$ abietis (17 insectes sur 569 piégés), mais ce champignon était présent sur tous les sites de piégeage, aussi bien en 1989 qu'en 1990. $P$ canum, isolé assez fréquemment sur les insectes piégés en forêt d'Orléans en 1989, n'a été isolé qu'une seule fois sur les insectes piégés dans cette même forêt en 1990. II était également présent sur les insectes provenant des Vosges ou du Massif central. $L$ procerum était le champignon le plus fréquemment associé à $H$ abietis. Aussi bien en 1989 qu'en 1990, le taux d'insectes vecteurs était de l'ordre de 40 à $45 \%$.

Les figures 1 et 2 rendent compte du pourcentage d'insectes vecteurs de $L$ procerum en fonction de l'année, du site et de la saison de piégeage. Des différences significatives sont constatées entre les taux de contamination des insectes piégés dans les différents sites. En 1989, en regroupant les captures effectuées dans les parcelles B140 et B339, d'une part, et dans les parcelles T376 et T325, d'autre part, on constate que le taux d'insectes vecteurs dans les parcelles boisées à proximité de taches de dépérissement était significativement supérieur à celui des insectes capturés en parcelles témoin. En forêt d'Orléans, en ne comparant que les 
Tableau I. Fréquences (en pourcentage de présence) des champignons associés à Hylobius abietis. Les nombres entre parenthèses représentent les limites des intervalles de confiance au seuil de $95 \%$.

Année de piégeage

1989

1990

Nombre d'insectes piégés

424

145

Pourcentage d'insectes vecteurs de :

Leptographium procerum

$44,6 \%$

$39,3 \%$

$(39,6-49,5)$

$(30,6-48,0)$

Pachnodium canum

$13,9 \%$

$(10,4-17,4)$

$2,8 \%$

$(0-6,1)$

Ophiostoma piliferum

$$
2,6 \%
$$

$(0,8-4,3)$

$4,1 \%$

$(0,2-8,1)$

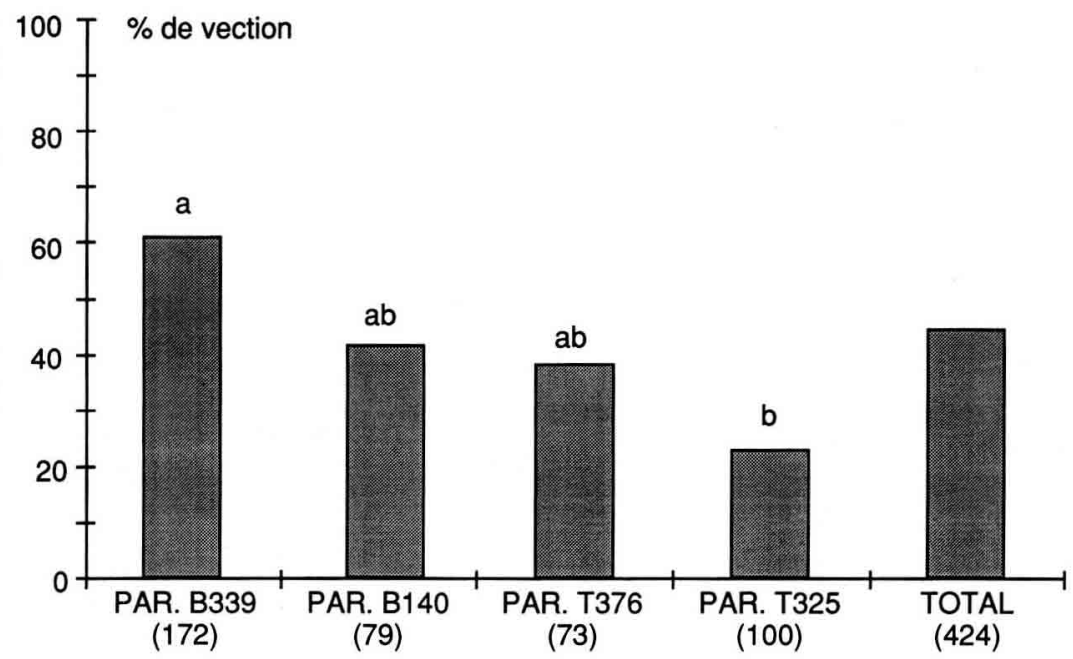

Fig 1. Pourcentages d'hylobes porteurs de Leptographium procerum en forêt d'Orléans en 1989. Le nombre d'insectes capturés par parcelle apparaît entre parenthèses. Les colonnes portant la même lettre ne diffèrent pas significativement entre elles au seuil de $95 \%$ (test $G$ ).

isolements réalisés du 19 avril au 15 mai, le taux d'insectes contaminés était significativement plus élevé en 1989 qu'en 1990. En été 1990, les insectes provenant des
Vosges présentaient un taux de contamination significativement plus élevé que celui des insectes prélevés en forêt d'Orléans. Le taux de contamination des hy- 


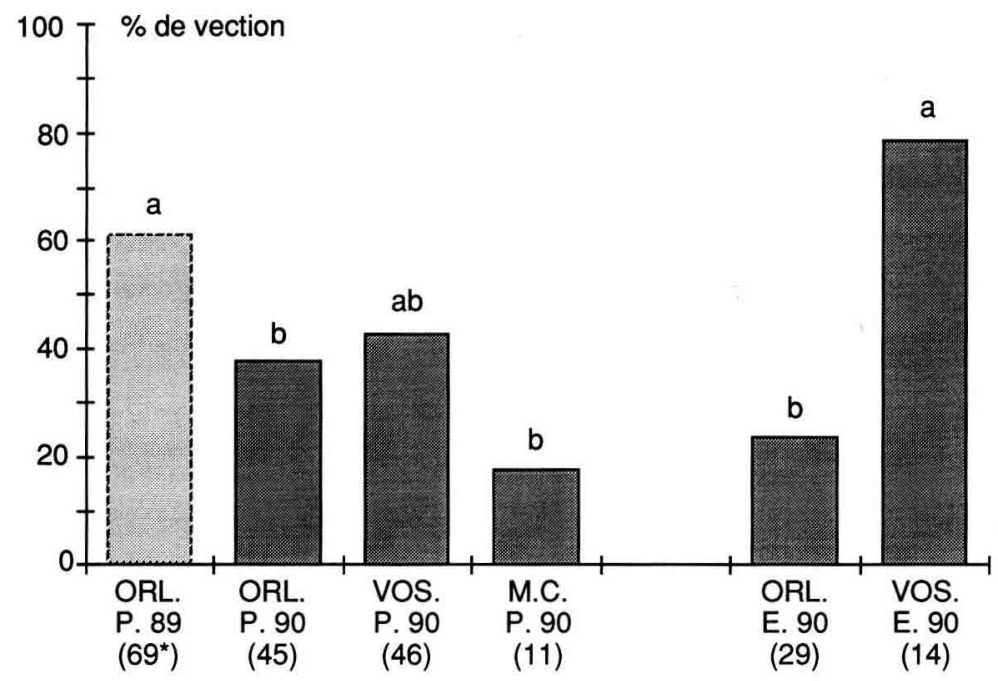

Fig 2. Pourcentages d'hylobes porteurs de Leptographium procerum dans 3 massifs, en fonction de la date de prélèvement. Légende : voir fig $1 ; O R L=$ Orléans; VOS $=$ Vosges; $M C=$ Massif central; $P$ : printemps; $E$ = été; * du 19 avril au 11 mai.

lobes n'est donc pas stable à la fois dans l'espace et dans le temps.

Le faible nombre d'insectes capturés certaines semaines en 1989 ne permet pas de discuter de l'évolution hebdomadaire du taux de contamination par parcelle. En regroupant les captures, on constate que la moyenne mobile hebdomadaire de périodicité 3 du taux de contamination a évolué de manière synchrone à la fois dans les deux coupes à blanc et dans les deux parcelles boisées, l'augmentation du taux de contamination semblant correspondre à des périodes humides, sa diminution à des périodes sèches (fig 3). Cependant, les insectes capturés dans les parcelles boisées présentaient un taux de contamination toujours plus élevé, la différence n'étant significative que de début mai à début juin, période particulièrement chaude en 1989.

\section{Insectes émergents}

\section{Observation en chambre humide des rondelles issues de rondins-pièges}

Des fructifications de $L$ procerum n'ont été observées qu'au niveau de plages d'aubier bleui, sur 8 rondelles provenant de rondins installés fin mai 1989. La proportion d'aubier bleui représentait plus de $50 \%$ de la surface de la section pour 2 rondelles; entre 20 et $50 \%$ pour 4 ; et moins de $20 \%$ pour les 2 dernières. Les flammes de bleuissement se répartissaient sur l'ensemble de la surface sans être particulièrement tangentes à la zone mise au contact du sol. Aucune fructification n'a été observée sur les rondelles provenant des rondins installés en 1990. 


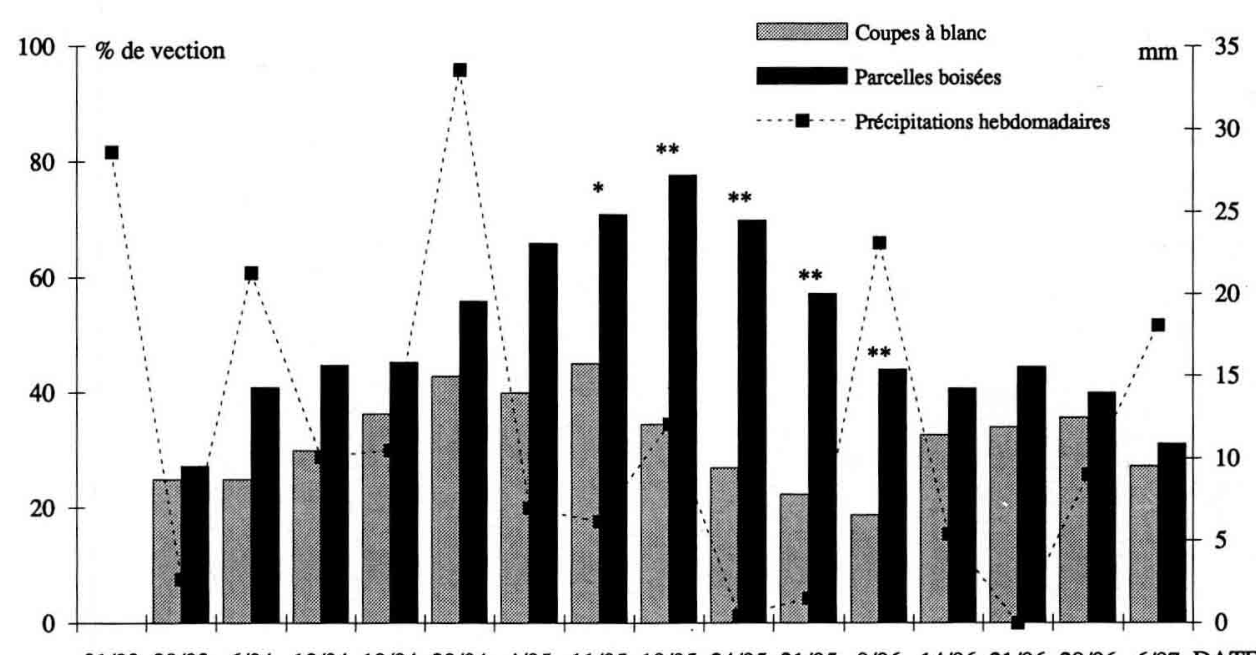

$\begin{array}{llllllllllllllll}21 / 03 & 29 / 03 & 6 / 04 & 12 / 04 & 19 / 04 & 29 / 04 & 4 / 05 & 11 / 05 & 18 / 05 & 24 / 05 & 31 / 05 & 8 / 06 & 14 / 06 & \text { DATE }\end{array}$

Fig 3. Évolution dans le temps du pourcentage d'insectes vecteurs de Leptographium procerum selon le type de parcelles. Chaque pourcentage correspond à une moyenne mobile de périodicité $3 .{ }^{*},{ }^{*}$ : différence significative au seuil de 5\%,1\% entre parcelles boisées et coupes à blanc.

\section{Champignons isolés à partir des émergents d'Hylobius abietis}

Entre le 5 juin et le 23 juillet 1990, 73 hylobes ont émergé des rondins installés au printemps 1989 (lot 1); 137 émergents ont été obtenus entre le 6 août et le 11 octobre 1990 à partir des rondins mis en place le 15 mars 1990 (lot 2). Le cycle des insectes du second lot n'a pas excédé 7 mois, contre au moins 12 mois pour les insectes du premier lot.

Sur les 4 champignons contaminant ces insectes émergents, 3 étaient identiques à ceux précédemment identifiés (tableau II). Seul Leptographium wingfieldii Morelet était nouveau. II n'était cependant associé qu'à un faible nombre d'individus du second lot ( 9 sur 137). $P$ canum n'était que très faiblement présent dans le second lot et $O$ piliferum était peu fréquent sur les
2 lots d'insectes émergents. $L$ procerum était présent dans le premier lot, sur environ la moitié des insectes émergents, ce qui est comparable au taux de vection trouvé en 1989 et 1990 . Il contaminait, en revanche, très peu d'insectes émergents du second lot.

Si on ne porte l'analyse que sur le lot 1 , $44 \%$ des mâles et $50 \%$ des femelles transportaient le champignon dès l'émergence. On constate par ailleurs une forte variation du poids des insectes émergents (entre $0,022 \mathrm{~g}$ et $0,186 \mathrm{~g}$ ). Une analyse de variance à 3 facteurs (rondins, sexe, présence ou absence de $L$ procerum) pour les insectes du lot 1, à 2 facteurs (rondins, sexe) pour les insectes du lot 2, fait apparaître un "effet rondin" significatif dans les 2 cas. Le sexe (lot 1 et 2 ) de l'hylobe et la contamination par $L$ procerum (lot 1 ) n'ont pas d'effet significatif sur le poids des émergents. 
Tableau II. Fréquence (en pourcentage de présence) des champignons associés aux hylobes émergents. Les nombres entre parenthèses représentent les limites des intervalles de confiance au seuil de $95 \%$.

Lot 1

Lot 2

Mise en place des rondins-pièges

Période d'émergence

Nombre d'insectes émergents

Pourcentage d'insectes vecteurs de :

Leptographium procerum

Pachnodium canum

Ophiostoma piliferum

Leptographium wingfieldii le 24 mai 1989

5 juin au 23 juillet 1990

73

$46,6 \%$

$(34,8-58,8)$

$0,0 \%$

$(0-4,9)$

$1,4 \%$

$(0,0-7,5)$

$0,0 \%$

$(0-4,9)$ le 15 mars 1990

6 août au 11 octobre 1990
137

$$
2,9 \%
$$

$(0,8-7,5)$

$1,5 \%$

$(0,2-5,3)$

$2,9 \%$

$(0,8-7,5)$

$6,6 \%$

\section{Isolement de champignons \\ sur les plants morts \\ suite aux morsures d'adultes d'hylobe}

À partir des 40 plants attaqués par l'hylobe en parcelle T325, $L$ procerum a été isolé 7 fois, soit de $17,5 \%$ des plants attaqués, et $P$ canum 3 fois, soit de $7,5 \%$ des plants attaqués.

\section{Inoculation de Leptographium procerum sur plants}

Les 12 plants inoculés sont morts 5 mois après l'apport du champignon sur les blessures, au début du printemps qui a suivi l'inoculation. Aucune mortalité n'a été enregistrée sur les plants blessés mais non inoculés. La figure 4 rend compte du nombre de réisolements de $L$ procerum aux différents niveaux. Si $L$ procerum est présent au niveau du collet sur les 12 plants inoculés, il semble coloniser beaucoup plus facilement la partie souterraine que la partie aérienne. À $10 \mathrm{~cm}$ du collet, il est absent de la tige dans tous les cas alors qu'il est présent dans les 3 quarts des cas au niveau de la partie racinaire. Les racines secondaires sont également envahies par $L$ procerum.

\section{INTERPRÉTATION ET DISCUSSION}

La faible fréquence de Pachnodium canum, d'Ophiostoma piliferum et de Leptographium wingfieldii sur les adultes d'hylobe, tant sur ceux piégés in situ que sur les émergents, laisse penser que ces isolements rélèvent plus de phénomènes fortuits que d'une réelle association. Leptographium procerum présente, en revanche, des taux élevés d'association avec Hylobius abietis dans tous les sites de prospection 


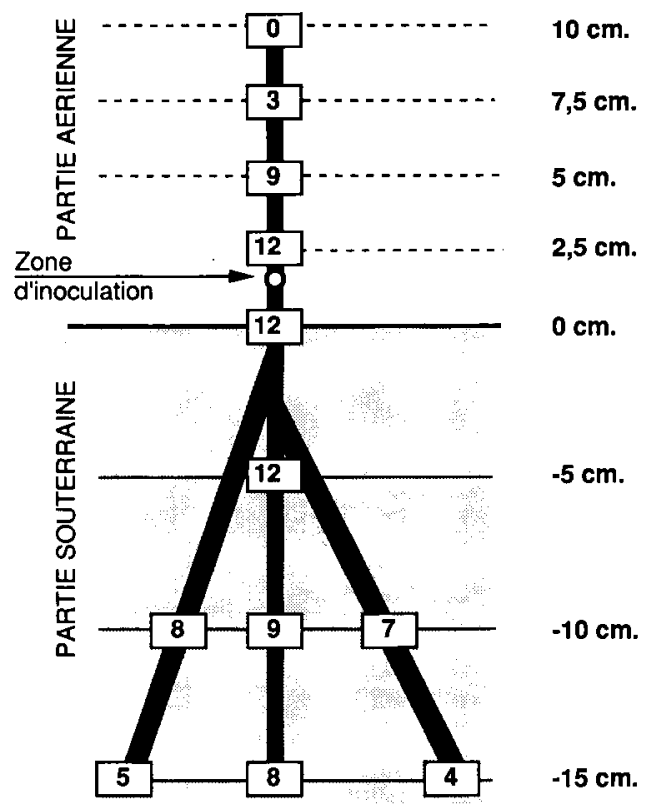

Fig 4. Nombre de réisolements de Leptographium procerum sur 12 plants morts, en fonction du niveau de prélèvement. $9=9$ réisolements réussis sur 12 tentés ( 1 par plant).

même dans les sites sans pin sylvestre comme dans le Massif central ou les Vosges (prélèvements d'été). Bien que les adultes puissent voler sur plusieurs dizaines de kilomètres (Solbreck, 1980), il est probable, compte tenu des pourcentages observés sur ces 2 sites, que certains insectes vecteurs étaient issus d'un ensouchement d'épicéa. Cette association n'est donc pas liée au développement larvaire de l'insecte dans un ensouchement de pin.

Swai et Hindal (1981), Lackner et Alexander (1984) ont fréquemment isolé $L$ procerum de la rhizosphère d'arbres dépérissants et très rarement de la rhizosphère d'arbres sains. Alexander et al (1988) concluent à la très faible capacité de survie des propagules de $L$ procerum dans le sol, hors de la rhizosphère d'arbres dépérissants. La contamination des rondinspièges sur le sol est donc très improbable. Dès lors, la seule voie de contamination possible des rondins demeure les insectes. Lieutier et al (1989) ne mentionnent pas ce champignon parmi ceux associés aux principaux scolytides des pins du massif d'Orléans. Ceux-ci, même lorsqu'ils se développent sur les mêmes rondins, ne peuvent donc pas être à l'origine de la contamination des hylobes. Dès lors, cette contamination ne peut provenir que des adultes parents qui inoculent probablement le champignon au niveau du site de développement de la génération suivante à l'occasion de la ponte.

En Europe, Kendrick (1962) mentionne la présence de $L$ procerum, isolé à partir de galeries de Pissodes pini prélevées sur Pinus sp en Suède. En Amérique du Nord, la présence simultanée de $L$ procerum et de galeries de curculionides au niveau des racines principales de pins dépérissants a conduit certains auteurs à soupçonner une association entre ce champignon et ces insectes (Webb et Alexander, 1982; Livingstone et Wingfield, 1982; Lackner et Alexander, 1982, 1984). Wingfield (1983) a isolé fréquemment $L$ procerum à partir de curculionides $(H$ radicis, $60 \%$; $H$ pales, entre 46 et $60 \% ; P$ picivorus, entre 30 et $70 \%$ ) et beaucoup moins fréquemment à partir du scolytide Dendroctonus valens. Lewis et Alexander (1986), dans des plantations de Pinus strobus avec ou sans dégâts provoqués par $L$ procerum, ont trouvé $64,2 \%$ des charançons et $0,76 \%$ des scolytes capturés vecteurs de $L$ procerum. Ces auteurs concluent que les charançons, dans ce cas $H$ pales et Pissodes sp, sont probablement les principaux vecteurs de $L$ procerum. $H$ abietis, principal représentant européen du genre Hylobius et qui possède une biologie proche de celles des hylobes nord-américains, transporte lui aussi $L$ procerum, indépendamment semble-t-il de 
tout dépérissement. Les taux de vection que nous avons pu trouver sont analogues à ceux cités pour $H$ radicus ou $H$ pales. Ces résultats confortent donc la notion d'association étroite proposée en Amérique du Nord avec les curculionides.

Dans le cas d'H abietis, nous avons pu constater d'importantes variations dans le temps et dans l'espace du taux d'insectes vecteurs. Les émergents du printemps 1990 , après une phase sous-corticale de 12 à 14 mois, présentent un taux de vection comparable à celui des adultes piégés durant cette période. Les émergents de l'été 1990, après 6 à 7 mois de développement sous-cortical, présentent un taux de vection très faible et les essais de réisolement du champignon à partir des rondelles n'ont jamais été positifs. On peut dès lors supposer que les conditions climatiques de l'été chaud et sec de 1990, qui ont permis un développement rapide des insectes, ne sont pas compatibles avec un développement saprophytique du champignon dans des rondins en cours de dessication à la surface du sol. Swai et Hindal (1981) rapportent d'ailleurs la difficulté d'isoler $L$ procerum de la rhizosphère d'arbres atteints durant les mois chauds. Le quasi-synchronisme de l'évolution des taux de contamination dans 4 parcelles, à l'intérieur d'un même massif, laisse par ailleurs supposer que cette contamination est sous la dépendance d'un facteur général externe. La pluviosité, en permettant une humidification suffisante des rondins, pourrait permettre le développement saprophytique du champignon. L'absence de précipitations en mai 1989 , durant une période chaude, s'accompagne d'ailleurs d'une baisse générale du taux de contamination. Le couvert forestier, en limitant la dessication des rondins, jouerait le même rôle, ce qui expliquerait le taux plus élevé constaté en parcelles boisées. Par ailleurs, la contamination par $L$ procerum ne semble avoir aucun effet sur le poids des émer- gents et ne gêne probablement pas le développement sous-cortical de l'insecte.

Le pouvoir pathogène de $L$ procerum a été discuté essentiellement en Amérique du Nord (voir les synthèses d'Alexander et al, 1988 , et de Wingfield et al, 1988). Les dépérissements européens dans lesquels ce champignon pourrait être impliqué sont peu nombreux (Morelet, 1986a, 1986b); seul Halambeck (1981) attribue à $L$ procerum des mortalités de Pinus strobus en Croatie.

Des trempages, avant repiquage, des racines de jeunes plants dans des suspensions de spores de $L$ procerum ont permis à Halambeck (1981) et à Lakner et Alexander $(1982,1983)$ de provoquer des mortalités et de conclure au pouvoir pathogène de ce champignon sur Pinus strobus ou sur $P$ taeda. En revanche, Lakner et Alexander (1982), Harrington et Cobb (1983) par insertion de fragments de bois contaminés dans des blessures pratiquées au niveau de la racine de jeunes plants, Wingfield (1983) par insertion de curedents contaminés dans la zone du collet, ne parviennent pas à provoquer des mortalités significatives. Le pouvoir pathogène de ce champignon apparaît comme étant fonction du mode d'inoculation.

L'apport d'implants mycéliens de $L$ procerum sur des blessures au collet, censées représenter des morsures de maturation d' $\mathrm{H}$ abietis, conduit à une mortalité de $100 \%$, alors que les blessures seules ne provoquent aucune mortalité. Ce mode d'inoculation conduit donc à des résultats contradictoires par rapport au mode d'inoculation par insertion de fragments de bois contaminés (Wingfield, 1983). Cela peut provenir des processus de colonisation de l'aubier par $L$ procerum. Horner et al (1987) ont montré que le champignon se propage dans les trachéides et dans les cellules parenchymateuses et conductrices des rayons ligneux, provoquant des accumulations de résine, le long des parois des trachéides qu'il colonise. Comme c'est le 
cas pour la plupart des agents de bleuissement du bois, la progression de $L$ procerum est plus importante dans le sens radial et longitudinal que dans le sens tangentiel (Alexander et al, 1988). L'inoculation au niveau de larges blessures pourrait donc conduire à une obturation suffisante des tissus conducteurs, induisant ainsi la mortalité, alors qu'un seul point d'inoculation latéral n'est probablement pas suffisante pour provoquer cette obstruction.

A l'occasion de ce type d'inoculation au collet, nous avons constaté un envahissement partiel du système racinaire par le champignon, lequel progresse peu vers la partie aérienne. Cela est conforme aux observations de Lewis et al (1987), qui avaient trouvé que la concentration des propagules de $L$ procerum dans la rhizosphère d'arbres atteints diminuait selon une distribution exponentielle négative au fur et à mesure de l'éloignement du collet.

Nous avons retrouvé $L$ procerum au collet de $17,5 \%$ des plants naturellement tués par $H$ abietis. Compte tenu de la probable impossibilité pour le champignon de contaminer les plants de proche en proche par le sol, $H$ abietis, seul insecte ayant attaqué les plants observés, apparaît être à l'origine de cette contamination par $L$ procerum.

$H$ abietis assure donc, vraisemblablement dans de nombreux massifs forestiers, le transport de $L$ procerum. Cet insecte est probablement à l'origine de la contamination de plants de pin sylvestre à l'occasion de ses repas de maturation au collet. Cela pourrait augmenter le taux de mortalité en provoquant le flétrissement des plants partiellement blessés mais qui auraient pu survivre à la seule morsure de l'insecte.

\section{REMERCIEMENTS}

Ce travail a été conduit au Laboratoire de zoologie forestière de I'INRA d'Orléans, grâce à un financement conjoint du ministère de l'Agriculture et de la Forêt et de la région Centre (Arbocentre). Nous tenons à remercier $\mathrm{CB}$ Malphettes et FX Saintonge du CEMAGREF, B Boutte et D Durmann du Département de la santé des forêts, pour leurs conseils ou leurs participations à la campagne de piégeage. Nous remercions également l'Office national des forêts (Direction régionale Centre, Service départemental du Loiret) pour la mise à disposition de parcelles d'études. Nous remercions enfin $F$ Lieutier et du $P$ du Merle pour la relecture qu'ils ont bien voulu effectuer du manuscrit.

\section{RÉFÉRENCES}

Alexander SA, Horner WE, Lewis KJ (1988) Leptographium procerum as a pathogen of pines. In: Leptographium Root Diseases on Conifers (Harrington TC, Cobb FW, eds). APS Press, St Paul, 97-112

Eidmann HH (1974) Hylobius Schönh. In: Die Forstchädlinge Mitteleuropas /I (W Schwenke, ed). Paul Parey, Hamburg-Berlin, 275-293

Francke-Grosmann $H$ (1967) Ectosymbiosis in wood-inhabiting insects. In: Symbiosis, vol 2 (Henry SM, ed). Academic Press, New York, 141-205

Furniss MM, Solheim H, Christiansen E (1990) Transmission of blue-stain fungi by lps typographus (Coleoptera : Scolytidae) in Norway spruce. Ann Entomol Soc Am 83, 712-716

Guiran $G$ de (1990) Les nématodes des pins (Bursaphelenchus spp). Problèmes biologiques, taxonomiques et épidémiologiques. $C R$ Acad Agri Fr 73, 13-20

Halambeck M (1981) Verticicladiella procera Kendrick uzrocnik venuca americkog borovca u kulturama cetinjaca. Zast Bilja 32, 313-323

Harrington TC (1981) Cycloheximide sensitivity as a taxonomic character in Ceratocystis. Mycologia 73, 1123-1129

Harrington TC, Cobb FW (1983) Pathogenicity of Leptographium and Verticicladiella spp isolated from roots of western north american conifers. Phytopathology 73, 596-599

Heritage S, Collins S, Evans HF (1989) A survey of damage by Hylobius abietis and Hylastes spp in Britain. In: Proceedings of a meeting of the IUFRO working group on insects affecting reforestation. Vancouver, 3-9 juillet 1988 (Alfaro RI, Glover SD, ed) 36-42 
Horner WE, Alexander SA, Lewis KJ (1987) CoIonization patterns of Verticicladiella procera in scots and eastern white pine and associated resin-soaking, reduced sapwood moisture content, and reduced needle water potential. Phytopathology 77, 557-560

Kendrick WB (1962) The Leptographium complex. Verticladiella Hughes. Can J Bot 40, 771-797

Lackner AL, Alexander SA (1982) Occurrence and pathogenicity of Verticladiella procera in Christmas tree plantations in Virginia. Plant Dis 66, 211-212

Lackner AL, Alexander SA (1983) Root disease and insect infestation of air-pollution sensitive Pinus strobus and studies of pathogenicity of Verticladiella procera. Plant Dis 67, 679-681

Lackner AL, Alexander SA (1984) Incidence and development of Verticicladiella procera in Virginia Christmas tree plantations. Plant Dis 68, 210-212

Langström B (1982) Abundance and seasonal activity of adult Hylobius weevils in reforestation areas during the first years following final felling. Commun Inst For Fenn 106, 1-23

Lanier GN, Peacock JW (1981) Vectors of the pathogen. In: Compendium of elm diseases (Stripes RJ, Campana RJ, eds). APS Press, St Paul, 14-16

Lewis KJ, Alexander SA (1986) Insects associated with the transmission of Verticladiella procera. Can J For Res 16, 1330-1333

Lewis KJ, Alexander SA, Horner WE (1987) Distribution and efficacy of propagules of Verticladiella procera in soil. Phytopathology 77 , 552-556

Lieutier F, Yart A, Garcia J, Ham MC, Morelet M, Levieux J (1989) Champignons phytopathogènes associés à deux coléoptères Scolytidae du pin sylvestre (Pinus sylvestris L) et étude préliminaire de leur agressivité envers I'hôte. Ann Sci For 46, 201-216
Livingstone WH, Wingfield MJ (1982) First report of Verticladiella procera on pines of Minnesota. Plant Dis 66, 260-261

McCall KA, Merril W (1980) Selective medium for Verticicladiella procera. Plant Dis 64, 277-278

Morelet M (1986a) Les Verticicladiella des pins en liaison avec les phénomènes de dépérissement. EPPO Bull 16, 473-477

Morelet M (1986b) À propos de Verticicladiella procera sur pin maritime (note rectificative). Ann de la SSNATV 38, 239-241

Piou D, Lieutier F (1989) Observations symptomatologiques et rôles possibles d'Ophiostoma minus Hedge (Ascomycètes: Ophiostomatales) et de Tomicus piniperda L (Coleoptera : Scolytidae) dans le dépérissement du pin sylvestre en forêt d'Orléans. Ann Sci For 46, 39-53

Scherrer B (1984) Biostatistique. Gaëtan Morin, Chicoutimi

Solbreck C (1980) Dispersal distances of migrating pine weevils, Hylobius abietis (Coleoptera:Curculionidae). Entomol Exp Appl 28, 123 131

Swai IS, Hindal DF (1981) Selective medium for recovering Verticladiella procera from soils and symptomatic white pines. Plant Dis 65, 963-965

Webb RS, Alexander SA (1982) Incidence of resin-soaked roots in subsoiled loblolly pine seed orchards on sandy soil. South J App/ 6 , 104-107

Wingfield MJ (1983) Association of Verticladiella procera and Leptographium terebrantis with insects in the Lake States. Can J For Res 13, 1238-1245

Wingfield MJ, Capretti P, Mac Kenzie M (1988) Leptographium spp as root pathogens of conifers. An international perspective. In: Leptographium Root Diseases on Conifers (Harrington TC, Cobb FW, eds). APS Press, St Paul, 113-128 